\title{
A REMARK ON REAL CHARACTERS OF COMPACT GROUPS
}

\section{FALKO LORENZ}

1. In this note we shall extend a theorem of Frobenius and Schur [1] on real characters of finite groups. We want also to suggest a simple proof for this result using only basic linear algebra.

Let $G$ be a compact group, and let $M$ be a finite-dimensional irreducible representation module for $G$ over the field $C$ of complex numbers. We denote by $\chi$ the corresponding character of $G . \chi$ is called realizable over $R$ (the field of real numbers), if there exists a $R G$ module $N$ such that the $C G$-module $N \otimes_{R} C$ is isomorphic to $M$. Clearly, a necessary condition for $\chi$ to be realizable over $R$ is that $\chi(g)$ be a real number for each $g$ in $G$. If the latter is the case we call $\chi$ real. The problem of course is to decide which real characters are also realizable over $R$. We define an invariant $c=c(\chi)$ of $\chi$ by setting $c=1$ if $\chi$ is realizable over $R, c=-1$ if $\chi$ is real but not realizable over $R$, and $c=0$ if $\chi$ is not real. Then we have the following criterion:

$$
c(\chi)=\int_{G} \chi\left(g^{2}\right) d g .
$$

Here $\int$ denotes the normalized Haar integral of the compact group $G$ (i.e. $\int_{G} d g=1$ ). Frobenius and Schur stated this theorem for the case of a finite group, where of course the integral on the right side of (1) is equal to the sum of all $\chi\left(g^{2}\right), g$ in $G$, divided by the order of $G$.

2. We consider the $C G$-module $M \otimes_{C} M$ to which the character $\chi^{2}$ belongs. We have

$$
M \otimes_{C} M=S(M) \oplus A(M)
$$

where $S(M)$ (resp. $A(M)$ ) denotes the $G$-submodule of symmetric (resp. antisymmetric) tensors of $M \otimes_{C} M$. Hence it follows for the corresponding characters

$$
\chi^{2}=\chi_{s}+\chi_{a}
$$

There is a simple relation between the values of $\chi_{s}$ and $\chi_{a}$, namely

$$
\chi_{*}=\chi_{a}(g)+\chi\left(g^{2}\right)
$$

(this is easily checked by taking a diagonal basis for the endomorphism of $M$ corresponding to $g$ ).

Received bv the editors June 2, 1968. 
Making use now of the orthogonality relations for $\chi$ and its conjugate $\bar{\chi}$ (defined by $\bar{\chi}(g)=\overline{\chi(g)}=\chi\left(g^{-1}\right)$ ) we see that $\int \chi^{2}(g) d g$ is equal to 1 if $\chi$ is real, and equal to 0 otherwise. This can also be expressed by saying that the tensors of $M \otimes_{C} M$ which are invariant under each $g$ in $G$ form a subspace of dimension 1 or 0 depending on whether $\chi$ is real or not. If we set $c^{\prime}=\int \chi\left(g^{2}\right) d g=\int \chi_{s}(g) d g-\int \chi_{a}(g) d g$ we conclude from (2) that $c^{\prime}$ can only be 0,1 , or -1 . Furthermore, $c^{\prime}=0$ is equivalent to $\chi$ being not real and $c^{\prime}$ is equal to 1 if and only if there is a symmetric tensor in $M \otimes_{C} M$ not equal to zero and invariant under $G$. Therefore (1) will be a consequence of the theorem below:

THEOREM. The following statements are equivalent:

(i) $\chi$ is realizable over $R$.

(ii) There exists a nonzero symmetric tensor in $M \otimes_{C} M$ which is invariant under $G$.

3. From now on let $\chi$ be real. In order to prove the theorem just stated we use the following fact which is generally known under the name of "Weyl's trick": There exists on $M$ a positive definite hermitian form $h$ which is invariant under $G$, i.e., $h(g x, g y)=h(x, y)$ for all $g$ in $G$ and all $x, y$ in $M$. (If $h^{\prime}$ is any positive definite hermitian form on $M$, define $h$ by $h(x, y)=\int_{G} h^{\prime}(g x, g y) d g$. Then $h$ does the trick.)

For a moment we consider also the dual space $M^{0}$ of $M$ endowed with its natural $G$-module structure: For every $f$ in $M^{0}$ and every $g$ in $G$ the element of $g f$ of $M^{0}$ is defined by $g f(x)=f\left(g^{-1} x\right)$ for all $x$ in $M$. The corresponding character is $\bar{\chi}$. Since $\chi$ is real we must have $M \cong M^{0}$. By looking at the resulting isomorphism $M \otimes_{C} M \cong M^{0} \otimes_{C} M^{0}$ $\cong\left(M \otimes_{C} M\right)^{0}$ we see that condition (ii) above is equivalent to

(ii') There exists a nonzero bilinear symmetric form $b$ on $M$ which is invariant under $G$, i.e. $b(g x, g y)=b(x, y)$ for all $g$ in $G$ and all $x, y$ in $M$.

We assume first that (ii') is satisfied. Since the form $h$ is nondegenerate there is a map $j: M \rightarrow M$ such that

$$
b(x, y)=h(x, j y) \quad \text { for all } x, y \text { in } M .
$$

Furthermore, $j$ is semilinear with respect to conjugation in $C$ and $j g x=g j x$ for all $g$ in $G$ and all $x$ in $M$. Then $j^{2}=j \circ j$ is an isomorphism of the $C G$-module $M$ and it follows from Schur's Lemma that $j^{2}$ $=c \operatorname{id}_{M}$ where $c$ is a complex number and $\operatorname{id}_{M}$ the identity map on $M$. We assert that $c$ is a real number greater than zero. To see this we set in (5) $y=j x$. Using the symmetry of $b$ we get $h\left(x, j^{2} x\right)=h(j x, j x)$ or $\bar{c} h(x, x)=h(j x, j x)$. Our assertion follows from the fact that $h$ is positive definite. Replacing now $j$ by $c^{-1 / 2} j$ (where $c^{-1 / 2}$ is the reciprocal of a 
square root of $c$ in $R$ ) we see that we can assume that $j^{2}=\mathrm{id}_{M}$. But then it follows that for $N=\{x ; j x=x\}$ we have $M=N \oplus i N=N \otimes_{R} C$, i.e. $\chi$ is realizable over $R$.

On the other hand, if $\chi$ is realizable over $R$, the $\operatorname{map} j=\mathrm{id}_{N} \otimes \sigma$, where $\sigma$ denotes conjugation in $C$, is a semilinear map of $N \otimes_{R} C$ with $j^{2}=\mathrm{id}_{M}$ and such that $j g x=g j x$ for all $g$ in $G$ and all $x$ in $M$. Then $b$ defined by $b(x, y)=h(x, j y)$ is a nonzero bilinear form on $M$ which is invariant under $G$, i.e. (ii') and hence (ii) is satisfied.

4. Let the group $G$ now be finite. It would be interesting to know if anything similar to Frobenius and Schur's criterion can be said for quadratic extensions $L / K$ other than $C / R$. There is one easy example which we want to mention here: Let $K$ be a real field (i.e. -1 is not a sum of squares in $K$ ) and suppose further that $K$ has the property that for every $a$ in $K$ either $a$ or $-a$ is a square in $K$. It follows that $K$ can be regarded as an ordered field whose positive elements are just the squares of $K$. There is one and only one quadratic extension $L$ of $K$ namely the field $L=K\left((-1)^{1 / 2}\right)$. Then, if we replace $R$ by $K$ and $C$ by $L$, the criterion of Frobenius and Schur remains valid also in this situation. The proof is completely analogous to the one given above in the case $K=R$ and $L=C$.

\section{REFERENCE}

1. G. Frobenius and I. Schur, Über die reellen Darstellungen der endlichen Gruppen, S.-B. Preuss. Akad. Wiss. (1906), 186-208.

Universität HeIdelberg, Germany 